Swarthmore College

Works

4-1-2003

\title{
Rapid Multiplexed Data Acquisition: Application To Three- Dimensional Magnetic Field Measurements In A Turbulent Laboratory Plasma
}

Matthew Joseph Landreman , '03

C. D. Cothran

Michael R. Brown

Swarthmore College,doc@swarthmore.edu

M. Kostora

J. T. Slough

Follow this and additional works at: https://works.swarthmore.edu/fac-physics

Part of the Physics Commons

Let us know how access to these works benefits you

\section{Recommended Citation}

Matthew Joseph Landreman , '03; C. D. Cothran; Michael R. Brown; M. Kostora; and J. T. Slough. (2003).

"Rapid Multiplexed Data Acquisition: Application To Three-Dimensional Magnetic Field Measurements In A Turbulent Laboratory Plasma". Review Of Scientific Instruments. Volume 74, Issue 4. 2361-2368. DOI: 10.1063/1.1544417

https://works.swarthmore.edu/fac-physics/96

This work is brought to you for free by Swarthmore College Libraries' Works. It has been accepted for inclusion in Physics \& Astronomy Faculty Works by an authorized administrator of Works. For more information, please contact myworks@swarthmore.edu. 


\section{AP $\mid \begin{aligned} & \text { Review of } \\ & \text { Scientific Instruments }\end{aligned}$}

Rapid multiplexed data acquisition: Application to three-dimensional magnetic field measurements in a turbulent laboratory plasma

M. Landreman, C. D. Cothran, M. R. Brown, M. Kostora, and J. T. Slough

Citation: Review of Scientific Instruments 74, 2361 (2003); doi: 10.1063/1.1544417

View online: http://dx.doi.org/10.1063/1.1544417

View Table of Contents: http://scitation.aip.org/content/aip/journal/rsi/74/4?ver=pdfcov

Published by the AIP Publishing

\section{Articles you may be interested in}

Probe measurements of the three-dimensional magnetic field structure in a rotating magnetic field sustained field-reversed configuration

Phys. Plasmas 21, 012502 (2014); 10.1063/1.4861131

High spatial resolution Hall sensor array for edge plasma magnetic field measurements

Rev. Sci. Instrum. 76, 093501 (2005); 10.1063/1.2018628

Development, tests, and data acquisition of the integrated system of internal sensors for RFX

Rev. Sci. Instrum. 75, 4338 (2004); 10.1063/1.1789624

Measurements of magnetic field fluctuations using an array of Hall detectors on the TEXTOR tokamak

Rev. Sci. Instrum. 73, 3482 (2002); 10.1063/1.1502018

Direct magnetic field measurement of flow dynamics in magnetized coaxial accelerator channels

Phys. Plasmas 4, 2820 (1997); 10.1063/1.872415

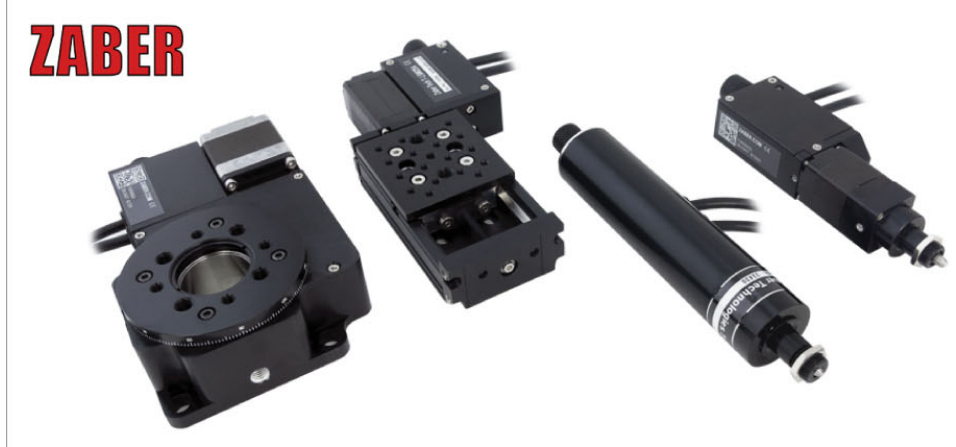

Automate your research applications with Zaber's line of high precision positioning devices.

Low cost. Built-in controllers. Simple to set up and easy to use. 


\title{
Rapid multiplexed data acquisition: Application to three-dimensional magnetic field measurements in a turbulent laboratory plasma
}

\author{
M. Landreman, ${ }^{\text {a) }}$ C. D. Cothran, and M. R. Brown \\ Department of Physics and Astronomy, Swarthmore College, Swarthmore, Pennsylvania 19081 \\ M. Kostora and J. T. Slough \\ Redmond Plasma Physics Laboratory, University of Washington, Seattle, Washington 98195
}

(Received 4 June 2002; accepted 16 December 2002)

\begin{abstract}
Multiplexing electronics have been constructed to reduce the cost of high-speed data acquisition at the Swarthmore Spheromak Experiment (SSX) and Redmond Plasma Physics Laboratory. An application of the system is described for a three-dimensional magnetic probe array designed to resolve magnetohydrodynamic time scale and ion inertial spatial scale structure of magnetic reconnection in a laboratory plasma at SSX. Multiplexing at $10 \mathrm{MHz}$ compresses 600 pick-up coil signals in the magnetic probe array into 75 digitizer channels. An external master timing system maintains synchronization of the multiplexers and digitizers. The complete system, calibrated and tested with Helmholtz, line current, and magnetofluid fields, reads out the entire $5 \times 5 \times 8$ probe array every $800 \mathrm{~ns}$ with an absolute accuracy of approximately $20 \mathrm{G}$, limited mainly by bit error. (C) 2003 American Institute of Physics. [DOI: 10.1063/1.1544417]
\end{abstract}

\section{INTRODUCTION}

In many experimental disciplines, cost often constrains the number of measurements that can be performed on a physical system. The cost of each digitizer channel is on the order of $\$ 1000.00$, so instruments with several hundred detectors are usually impractical. For experiments on a $1 \mathrm{~ms}$ or longer timescale, one possible solution is to insert a layer of multiplexing electronics to make each digitizer channel record signals from a number of individual detectors, typically 4,8 , or 16 , therefore reducing the cost of the total system by a similar factor. Multiplexing techniques in a variety of contexts have been described on several occasions in this journal, ${ }^{1,2}$ and commercial "data logger" devices are available for such slow tasks as recording the temperature at many locations. However, no commercial systems presently exist for $\mathrm{MHz}$ or faster applications. Recently, cost reductions from the commercialization of video multiplexing technology now make high-speed general purpose multiplexing data acquisition systems possible.

The Swarthmore Spheromak Experiment $(\mathrm{SSX})^{3}$ has recently completed construction, calibration, and testing of a 600 coil magnetic probe array and an 8:1 multiplexing data acquisition system designed for measurements of the threedimensional (3D) structure of magnetic reconnection in a magnetohydrodynamic (MHD) laboratory plasma. The probe array measures the three components of the magnetic field at each point of a $5 \times 5 \times 8$ lattice with $2 \mathrm{~cm}$ resolution, comparable to the ion inertial scale in SSX plasmas. The multiplexed data acquisition system samples all 600 signals every $800 \mathrm{~ns}$, corresponding to a time resolution sufficient to capture the fastest characteristic MHD time scale of interest. The

\footnotetext{
a) Author to whom correspondence should be addressed; electronic mail: mlandre1@swarthmore.edu
}

core of the multiplexing electronics was developed in collaboration with the Redmond Plasma Physics Laboratory (RPPL) at the University of Washington, although slightly different designs were produced for the unique needs of each institution. Our laboratories have each used the multiplexing electronics for a variety of diagnostic measurements. For brevity, this paper documents only the magnetic probe array implemented at SSX.

This article is divided into seven sections. Following this Introduction, Sec. II describes the motivation and design goals for developing an instrument to measure the 3D magnetic structure of reconnection. Details of the custom multiplexer electronics are given in Sec. III. The data acquisition system, including the synchronization of the multiplexers and digitizers, is described in Sec. IV. Section V discusses the construction of the magnetic probe array. Finally, results of calibration and testing of the complete system are described in Sec. VI and conclusions follow.

\section{MOTIVATION AND DESIGN GOALS}

Magnetic reconnection occurs when two bodies of highly conductive plasma bearing oppositely directed embedded magnetic fields merge. ${ }^{3,4}$ It is a ubiquitous phenomenon, 5 occurring in the magnetosphere, on the sun, and in many other astrophysical systems, as well as in fusion plasmas. Various properties of reconnection have been studied in laboratory plasmas, including heating, bulk flows, energetic particles, and magnetic structure. ${ }^{6}$ These studies, however, have all been conducted in either cylindrical or linear "slab" geometries where the behavior along one dimension was assumed to be uninteresting. ${ }^{6}$ Magnetic structure was measured with at most two-dimensional (2D) arrays of magnetic probes. Results indicated $X-, Y$-, and $O$-shaped reconnection topologies, ${ }^{7,8}$ and the $2 \mathrm{D}$ assumption justified analysis with flux functions to infer reconnection rates. 
Although some inherently 3D effects were studied, such as differences in reconnection rates for co- and counterhelicity toroidal plasma ${ }^{9}$ and ion heating by the "slingshot" effect from newly reconnected field lines, ${ }^{10}$ any interesting 3D magnetic structures were either present but unmeasured with the $2 \mathrm{D}$ probe arrays, or possibly suppressed by the global geometric constraints. In fact, both simulation ${ }^{11}$ and analytical ${ }^{12}$ calculations recently predict that the Hall term in the generalized Ohm's law should generate unique 3D magnetic structures within an ion inertial scale of the reconnection layer. It is therefore an outstanding experimental problem, and the motivation for the instrument described in this article, to examine the full 3D magnetic structure of reconnection.

The most straightforward instrument for such a measurement is a 3D array of magnetic probes (inductive pick-up coils). As with all plasma diagnostics, this probe array should have as small an effect as possible on the reconnecting plasmas. This means it should present a minimal restriction to flows, magnetic fields should penetrate it on time scales faster than the MHD time scales of interest, and it must withstand the aggressive environment of a hot, dense plasma. The probe spacing should be comparable to the relevant physical scale, in this case the ion inertial scale. The number of probes should be sufficient to reach the bulk inflow and outflow regions of the reconnection zone, and there should be a similar number in the out-of-plane direction. With just a few in each direction, the total number of probes quickly numbers in the hundreds. Apart from the number, however, these considerations are not extraordinary and conventional magnetic probe designs can be applied.

Other than an array of probes, a notable alternate solution is to map out $3 \mathrm{D}$ structures with a single movable probe by averaging over thousands of shots in a highly reproducible plasma, as was done at UCLA ${ }^{13}$ to study current sheet instabilities in an electron-MHD regime (unmagnetized ions). This approach certainly minimizes the perturbation to the plasma and requires the simplest data acquisition system. However, the averaging limits the dynamical information available with such an approach. Correlated magnetic fluctuations throughout the volume of the reconnection region, for instance, are lost. Furthermore, the reproducibility of the plasma from shot to shot is difficult to achieve for plasmas fully in the MHD regime.

Since a measurement that captures the full dynamical content of the 3D magnetic structure of reconnecting plasmas requires simultaneous (or nearly so) readout of hundreds of magnetic probes, the dominant design consideration is the cost of the data acquisition system. Fortunately, the fastest MHD time scale in SSX plasmas, a few microseconds, is very slow compared to the sampling rate of most digitizers, so a multiplexing data acquisition system is a practical solution. No commercial multiplexers operate at this speed, however, so custom electronics are necessary. This circuitry can be easily included with the integrating electronics necessary to reprocess the induced signals from each pick-up coil in the probe array, thereby reducing the overhead for this application.

\section{MULTIPLEXER BOARDS}

Figure 1 displays the circuit design schematic of the multiplexing electronics. Two slightly different sets of boards - one for SSX and one for RPPL-were produced concurrently, and the differences will be described below. The design was sufficiently complicated and the production scale sufficiently large that commercial contractors were hired for fabrication ${ }^{14}$ and assembly. ${ }^{15}$

Each circuit board accepts three sets of eight input signals. Each set is multiplexed 8:1 into a single output channel, for a total of three multiplexed output signals. There are also two TTL timing signals for each board, a clock and a gate. Although RPPL has successfully operated a prototype board at $20 \mathrm{MHz}$, these boards are designed to operate with a 10 $\mathrm{MHz}$ clock, which yields a multiplexed acquisition rate of 1.25 MHz. The circuit board design is divided into three functional units: input buffers (24 total), the multiplexer integrated circuits (ICs) (3), and a single binary counter for addressing.

Input signals are first terminated in $50 \Omega$ resistors. The input buffers for the SSX magnetic probe array application are active integrators with a $100 \mu$ s time constant, while the design for RPPL was easily modified to instead perform amplification on the inputs before multiplexing. This flexibility was an important consideration in the design to maximize the functionality of these circuit boards. The Analog Devices OP467 quad op-amp is specifically designed for high-speed instrumentation applications (28 MHz unity gain bandwidth). There is a $1 \mathrm{M} \Omega$ bleed resistor in parallel with the $10 \mathrm{nF}$ integrating capacitor to reset the integrator between shots (this $10 \mathrm{~ms}$ time constant is much longer than the 100-200 $\mu$ s duration of both SSX and RPPL plasmas). The $\pm 0.3-0.5$ $\mathrm{mV}$ typical input voltage offset for the OP467 leads to a $\pm 30-50 \mathrm{mV} \mathrm{dc}$ baseline offset at the integrator output.

There is one eight-channel multiplexer for each of the three sets of eight integrated signals. The Maxim MAX440 high-speed video multiplexer/amplifier was selected for this application, and the design follows the typical operating circuit suggested in the data sheet. The switching time for this multiplexer is $15-25 \mathrm{~ns}$. The amplifier stage has a $160 \mathrm{MHz}$ bandwidth and is designed to drive coaxial (50 or $75 \Omega$ ) loads. Despite the excellent bandwidth, the limiting characteristic for this application is its $65 \mathrm{~ns}$ settling time (to $0.1 \%$ ). External resistors set the gain of the amplifier stage to 2 . Bypass capacitors and a dedicated ground plane are used to maximize the ac performance of these multiplexers.

The clock and gate inputs to the circuit board control the 4 bit 74ALS161 counter which is used to address the multiplexers. This counter is inhibited while the gate is held high, and all 4 bits are low (address zero). While the gate is pulled low, each rising edge of the clock increments the counter. Only the lowest three bits of the counter are necessary to address the eight multiplexer channels. All three multiplexers share the same address lines. Clocking the counter at 10 $\mathrm{MHz}$ while the gate is pulled low therefore causes each multiplexer to switch through its eight input signals in sequence, with each input spending $100 \mathrm{~ns}$ at the multiplexer output. However, after accounting for the duration of the switching 


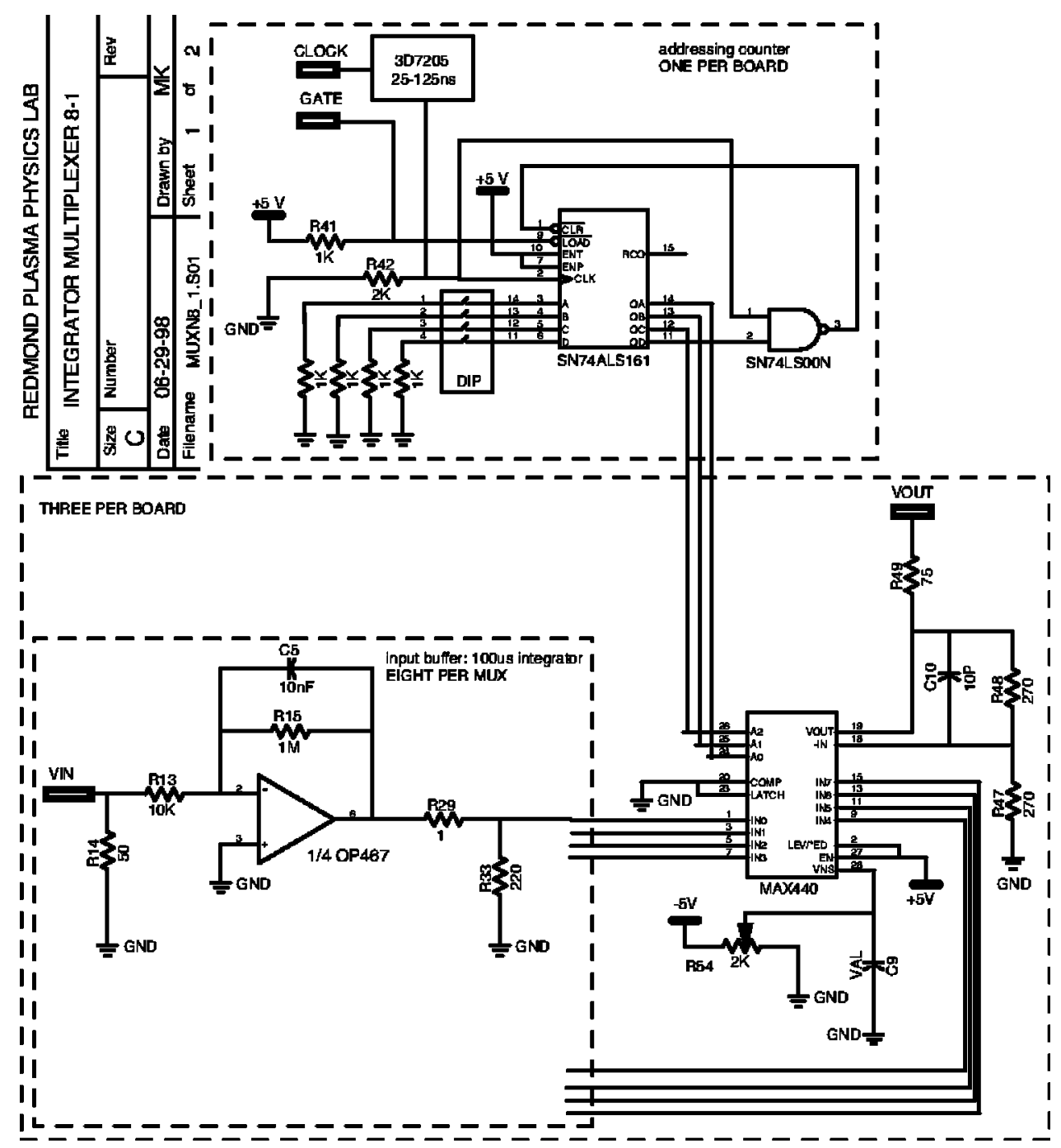

FIG. 1. Circuit board design developed at RPPL, consisting of three units of $8: 1$ buffering/multiplexing. There are a total of 24 input buffers (100 $\mu$ s integrators for this application), three multiplexers, and one addressing counter per board.

noise and the settling time, the actual time interval over which the output accurately reflects the input signal can be as little as $20 \mathrm{~ns}$.

The boards produced for RPPL included a Data Delay Devices, Inc. 3D7205 five-tap fixed delay chip to introduce a delay on the clock in multiples of $25 \mathrm{~ns}$. For the implementation at SSX, this feature was disabled. A marker pulse, generated by a NAND operation on the most significant bit of the counter with the clock, was also included as a diagnostic signal to help synchronize multiple boards.

\section{MULTIPLEXING DATA ACQUISITION SYSTEM}

Figure 2 illustrates the multiplexing data acquisition system. Operating with a $10 \mathrm{MHz}$ clock, this system samples all 600 probe array signals every $800 \mathrm{~ns}$ in 75 digitizer channels. There are three main subsystems: the 25 multiplexer boards, 10 eight-channel digitizer modules, and a synchronization and fan-out system for the clock and gate timing signals.

Synchronization of the clock and gate signals for all of the multiplexer boards and digitizer modules is the trickiest aspect of this acquisition system. Each digitizer module accepts signals from up to four different multiplexer boards, and, conversely, up to two different digitizer modules acquire signals from the same multiplexer board. It is by far simplest, if not essential, to have all of the multiplexers operating synchronously, and all of the digitizers sampling synchronously, while allowing for an overall delay between these two subsystems. It is found that the timing window for successful operation is $20-40 \mathrm{~ns}$, as described above. Care must also be taken to keep signal cable lengths the same to less than a few nanoseconds.

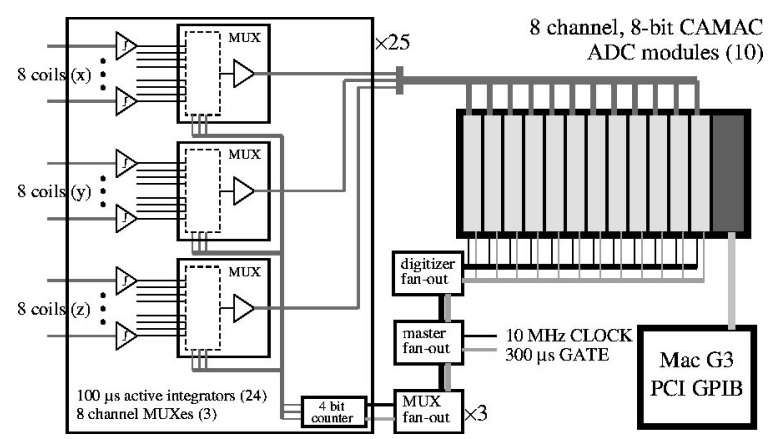

FIG. 2. Block diagram of the multiplexing data acquisition system consisting of 25 multiplexer boards, ten digitizer modules, and five clock and gate fan-out boards. 
(a)

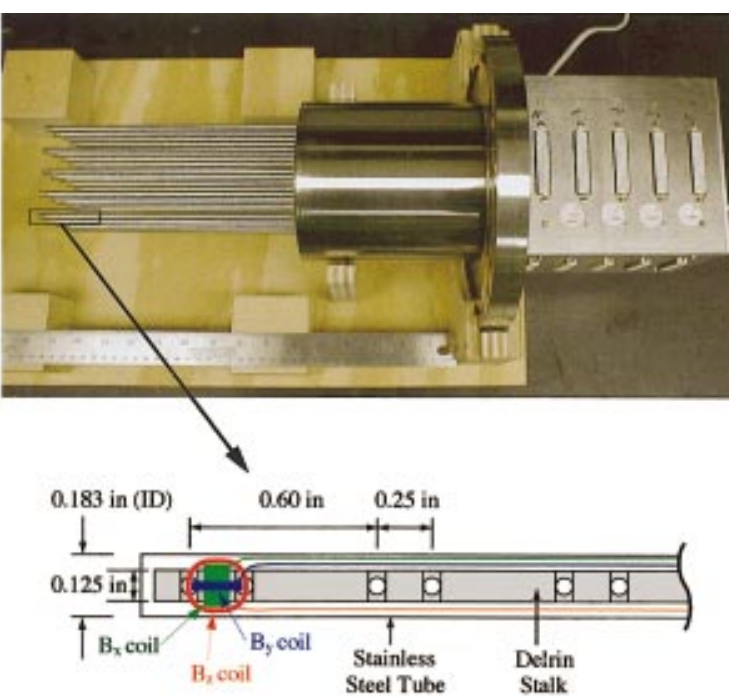

FIG. 3. (Color) A photograph (a) of the 3D magnetic probe array, and a cut-away sketch (b) of a probe-stalk.

At SSX, a function generator provides the master clock, and the master gate is derived from the SSX trigger. These master signals are distributed to the multiplexers and digitizers with a set of fan-out boards custom built using standard ICs. The chip-to-chip variation in the internal signal propagation delays was found to be small (less than $5 \mathrm{~ns}$ throughout the whole system). There is a master fan-out board, three fan-out boards for each rack of multiplexers, and one fan-out board for the ten digitizers. The master fan-out board first uses a flip-flop to synchronize the master gate with the master clock (they are initially asynchronous). The overall delay between the multiplexer boards and the digitizer modules is controlled on the digitizer fan-out board with two delay chips which allow the clock and gate inputs to be delayed in increments of $25 \mathrm{~ns}$. Cable delay is used for fine adjustment. RPPL built a completely different fan-out and synchronization system with commercial CAMAC and NIM modules for their applications.

The digitizer modules are all DSP Technology, Inc. model 2028 eight bit CAMAC digitizers. Each channel has a $50 \Omega$ input, a $10 \mathrm{MHz}$ analog to digital converter with \pm 256 $\mathrm{mV}$ range, programmable offset and attenuation, and a 32 kbyte memory. At unity attenuation, the bit resolution is 2 $\mathrm{mV}$, which determines the ultimate resolution for the magnetic field measurements. As described above, these modules are externally clocked and gated in this application. A Macintosh G3 computer running LABVIEW communicates via general purpose interface bus with the crate controller to download the data stored in the module memories after each shot. A straightforward software algorithm performs the demultiplexing to extract the waveform for each of the 600 signals.

\section{3D MAGNETIC PROBE ARRAY}

Figure 3(a) presents a photograph of the 3D magnetic probe array constructed at SSX. The probe housing consists of 25 thin wall (0.010 in.), 0.203 in. outer diameter stainless steel tubes welded onto a 10 in. conflat flange. These tubes are arranged in a $5 \times 5$ array with uniform 0.75 in. spacing in the coordinates $y$ and $z$. Inside each tube is a probe-stalk consisting of 24 coils wound on a 0.125 in. diam Delrin rod. A triplet of orthogonal coils is located at each of eight positions in the coordinate $x$ along the rod. The first six of the eight positions are spaced by 0.6 in., and the last two by 1.5 in. The 600 coils in this instrument, therefore, are grouped as triples on a $5 \times 5 \times 8$ lattice in $y, z$, and $x$, respectively.

Figure 3(b) illustrates one complete triplet of coils in a cutaway view of a single probe-stalk. The $\hat{\mathbf{x}}$ oriented coils, wound around the circumference of the rod, each have ten turns with an area of $8 \mathrm{~mm}^{2}$. The $\hat{\mathbf{y}}$ and $\hat{\mathbf{z}}$ oriented coils each have five turns with an area of $20 \mathrm{~mm}^{2}$. For these rectangularly shaped coils, wire is threaded through pairs of 0.25 in. spaced holes machined into the Delrin rod at each of the eight probe positions. All coils use 34AWG magnet wire with polythermaleze enamel. After each coil is wound, the ends of the remaining wire are made into a twisted pair, and all 24 twisted pairs from each probe-stalk are soldered to a 50-pin D-type connector (two pins are unused for each connector).

To prevent abrasion while being inserted into the tubing, each probe-stalk is wrapped with teflon tape. Alignment pins at the end of each Delrin rod ensure that all probe-stalks have been not only inserted to the same depth in each of the tubes, but also rotated to the same alignment. A breakout box for the 25 D-type connectors is mechanically secured to the conflat flange of the probe housing, but is electrically isolated since the flange is tied to machine ground once the probe array is installed into SSX.

There is one multiplexer board for each probe-stalk. The 24 signals available at each 50-pin connector on the probe array are carried on individually shielded twisted pairs bundled in a double-shielded cable (the gray cables seen in the photos in Figs. 6 and 8) to the input channels of each multiplexer board. The three multiplexing units on each board process the three sets of eight signals from the $\hat{\mathbf{x}}, \hat{\mathbf{y}}$, and $\hat{\mathbf{z}}$ oriented coils.

The eight raw signals for the $\hat{\mathbf{z}}$ coils of one probe-stalk which are multiplexed into a single digitizer channel are shown in Fig. 4. The individual offsets (originating in each integrator channel) have been subtracted from this data. Following the signals from the integrators, through the multiplexer amplifier, and into the terminating resistor in the digitizer channel, a straightforward calculation assuming ideal electronics and perfectly wound coils gives a sensitivity at the digitizer input of $12 \mathrm{G} / \mathrm{mV}$ for the $\hat{\mathbf{x}}$ oriented coils and 16 $\mathrm{G} / \mathrm{mV}$ for the $\hat{\mathbf{y}}$ and $\hat{\mathbf{z}}$ oriented coils of the probe array. The rms error corresponding to the $2 \mathrm{mV}$ bit resolution therefore sets the minimum achievable magnetic field measurement error for the probe array to be 8 and $10 \mathrm{G}$, respectively (assuming values distributed uniformly over a $2 \mathrm{mV}$ rectangular bin).

Figure 5 shows a sketch of the Swarthmore Spheromak Experiment $^{3}$ and two views of the 3D magnetic field measured with the probe array corresponding to the time $t$ $=64 \mu \mathrm{s}$ in Fig. 4. SSX produces spheromaks with coaxial magnetized plasma guns, one on each end of the cylindrical chamber. Spheromaks of opposite helicity are used for reconnection studies. The spheromaks relax inside separate, cylin- 


\section{$\mathrm{mBz}$}



FIG. 4. An example raw data set showing the acquisition of signals from eight different coils in one digitizer channel during a magnetofluid measurement.

drical copper flux conservers $(R=25 \mathrm{~cm})$, and reconnect across two sector-shaped slots cut in the back-to-back flux conserver walls at the midplane. The 3D magnetic probe array is inserted into the vacuum chamber at the midplane to measure the structure of the magnetic field in the reconnection region defined by the slots. The spacing in $x$ of the probe array lattice is selected to provide a high density of probes


FIG. 5. Sketch of the Swarthmore Spheromak Experiment and two views of the magnetic probe array data. Plasma guns produce spheromaks on either end of the cylindrical device. A cartoon of the poloidal and toroidal field components in the bulk spheromaks is shown for this counter-helicity configuration. The 3D magnetic probe array is inserted into the vacuum chamber at the midplane and measures the magnetic field structure in the region where the two spheromaks reconnect. The measured fields are shown in two orthogonal projections. The largest fields shown are approximately $800 \mathrm{G}$. For clarity, facing planes of data are dark, others are gray.
$6 \times 5 \times 5$ in the region of interest near the slots and a lower density $2 \times 5 \times 5$ nearer to the flux conserver walls. The data shown in this figure have been calibrated according to the procedure described in the next section.

Typical characteristics ${ }^{3}$ for the spheromak bulk magnetofluid are temperature $T \sim 20 \mathrm{eV}$, density $n$ $\sim 10^{13-14} / \mathrm{cm}^{3}$, and field strength $B \sim 500 \mathrm{G}$. Peak fields reach $1-2 \mathrm{kG}$. The Alfvén speed is $v_{A} \sim 10 \mathrm{~cm} / \mu \mathrm{s}$, so the crossing time is $\tau_{A} \sim 1-2 \mu \mathrm{s}$. This MHD time scale is slow compared to the penetration time $\tau \sim 0.1 \mu \mathrm{s}$ for magnetic fields to soak through the thin wall stainless steel tubing of the 3D probe array. The 100-200 $\mu$ s lifetime of these spheromaks is also short enough that stainless steel is an acceptable material to be introduced into the plasma.

\section{CALIBRATION AND TEST FIELD MEASUREMENTS}

The simple method, suggested in the previous section, of applying the 12 and $16 \mathrm{G} / \mathrm{mV}$ ideal sensitivities to the offsetsubtracted raw signals is not an adequate calibration procedure. There are a number of sources of error in the construction of the probe array that must be corrected with a more sophisticated calibration procedure. The sensitivities, for example, vary systematically by as much as $20 \%$ across the 600 channels, depending upon the precision of the electronic components, variations in the area enclosed by each coil, and possibly also occasional errors in the number of turns wound per coil. Furthermore, each triplet of coils may not be completely orthogonal and may not be exactly oriented along the $\hat{\mathbf{x}}, \hat{\mathbf{y}}$, and $\hat{\mathbf{z}}$ directions. The signals $\left(V_{x^{\prime}}, V_{y^{\prime}}, V_{z^{\prime}}\right)$ from each coil triplet in general represent the projection of the magnetic field along a linearly independent, but not necessarily orthogonal, set of directions $\hat{\mathbf{x}}^{\prime}, \hat{\mathbf{y}}^{\prime}$, and $\hat{\mathbf{z}}^{\prime}$. To extract the field components along the orthogonal directions, therefore, a linear transformation must be performed simultaneously on all three signals of each coil triplet. Rather than a scalar sensitivity for each coil, the calibration procedure must determine a $3 \times 3$ matrix $C$ such that $\mathbf{B}=C \mathbf{V}$ for each coil triplet both to balance the response of each channel of electronics as well as to eliminate the residual coil misalignments.

The uniform field of a $12 \mathrm{in.} \mathrm{diam} \mathrm{Helmholtz} \mathrm{pair} \mathrm{was}$ used for calibration, as shown in Fig. 6. When pulsed, these coils produced a peak field of approximately $1500 \mathrm{G}$, comparable to the maximum expected field strengths in SSX. Data were taken with the pair oriented in the $\hat{\mathbf{x}}, \hat{\mathbf{y}}$, and $\hat{\mathbf{z}}$ directions. Separately, a Rogowski coil measurement of the current in the Helmholtz pair was also digitized for each shot, and these data were used to compute the field produced at the position of each coil triplet in the probe array. The computed field components $\mathbf{B}$ and the corresponding offset subtracted signals $\mathbf{V}$ measured for these three shots with the Helmholtz pair determine all nine unknowns in the calibration matrix $C$ for each coil triplet.

The uncertainties in the computed fields and the errors in the measured signals determine the accuracy of these calibration matrices. The contributing uncertainties in the computed fields include nonideal effects in the construction of the Helmholtz pair such as the alignment and positioning of the two coils, error fields from the current input and return, and 
(a)

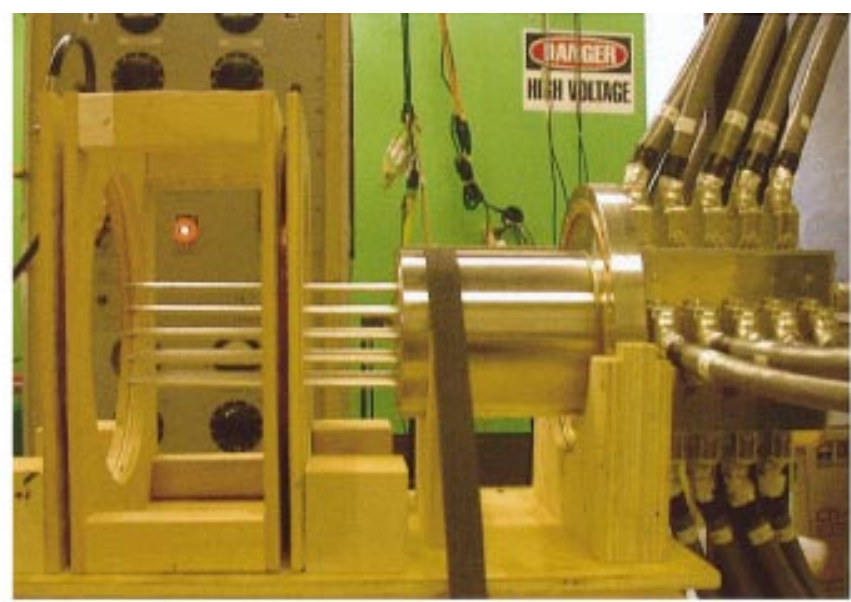

(b)

FIG. 6. (Color) A photograph (a) of the probe array during calibration with a Helmholtz pair, and a visualization (b) of the measured vector field after calibration.

uncertainties in the position of each coil triplet relative to the Helmholtz coordinate system. These effects, however, contribute negligibly to the total calibration error, primarily due to the nature of uniform field region. The dominant error from the calculated fields comes from the $2 \%$ accuracy of the Rogowski coil measurements. This source of error affects the normalization uncertainty of all calibration matrices equally. The contribution from the measured signals is set by the 2 $\mathrm{mV}$ digitizer accuracy. To minimize this contribution to the calibration error, the calibration is performed with data averaged over ten time steps at the peak of the Helmholtz field.

Based on these sources of error, the total uncertainty in the calibration matrices is estimated to be less than $0.5 \%$, with an overall normalization error of $2 \%$. After this calibration procedure is complete, the error on the magnetic field measurements $\mathbf{B}=C \mathbf{V}$ depend upon these errors in the calibration matrices $C$ and the bit error in the measured signals $\mathbf{V}$ in quadrature; symbolically this can be written loosely as $(\delta B)^{2}=[(\delta C) V]^{2}+(C \delta V)^{2}$. However, since the calibration matrices are very well determined compared to any single time-step measurement of $\mathbf{V}$, the error in the probe array magnetic field measurements is at best given by the $8-10 \mathrm{G}$ resolution derived in the previous section based on the bit error.

Test shots with the Helmholtz field after calibration are useful in verifying this result for the resolution of the probe array. Since $\boldsymbol{\nabla} \cdot \mathbf{B} \equiv 0$ for all magnetic fields, and $\boldsymbol{\nabla} \times \mathbf{B}=0$ for vacuum fields, the mean square deviation of $\boldsymbol{\nabla} \cdot \mathbf{B}$ and $|\nabla \times \mathbf{B}|$ from zero, computed with the lattice of probe array data, directly reflects the errors in the magnetic field measurements. The errors on the derivatives in these quantities, computed as finite differences of the data at two lattice points, in general contain the errors in the two measurements, uncertainties in the lattice coordinates, and corrections for any curvature in the fields. For Helmholtz fields, the latter two are negligible. The error on any finite difference derivative $D B=[B(x+L)-B(x)] / L$ in this case is simply $(\delta D B)^{2}=2[(\delta B) / L]^{2}$, assuming the errors at the two lattice points are the same and uncorrelated. Figure 7 shows the average measurement error $\delta B$ for the probe array as con- cluded in this manner from the mean square deviation of $\boldsymbol{\nabla} \cdot \mathbf{B}$ and $|\boldsymbol{\nabla} \times \mathbf{B}|$ from zero for the duration of a Helmholtz shot. Since $\delta B$ contains no hint of the wave form of the current driven through the Helmholtz coils, the contribution to $\delta B$ from errors in the calibration matrices (see above) are negligible, as expected. The constant level of 10-20 G confirms the assertion that the resolution of the probe array mea-
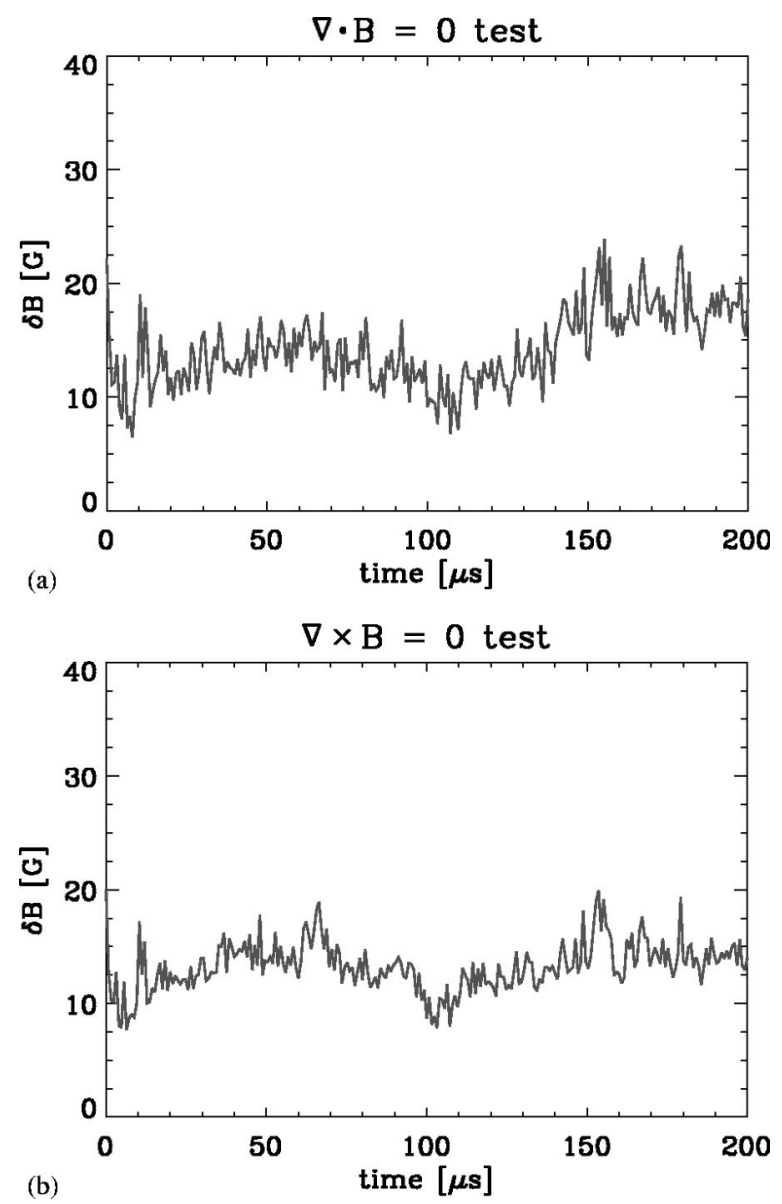

FIG. 7. Average magnetic field measurement error $\delta B$ derived from the mean square deviation from zero of: (a) $\boldsymbol{\nabla} \cdot \mathbf{B}$ and (b) $\boldsymbol{\nabla} \times \mathbf{B}$ for Helmholtz fields. 
(a)

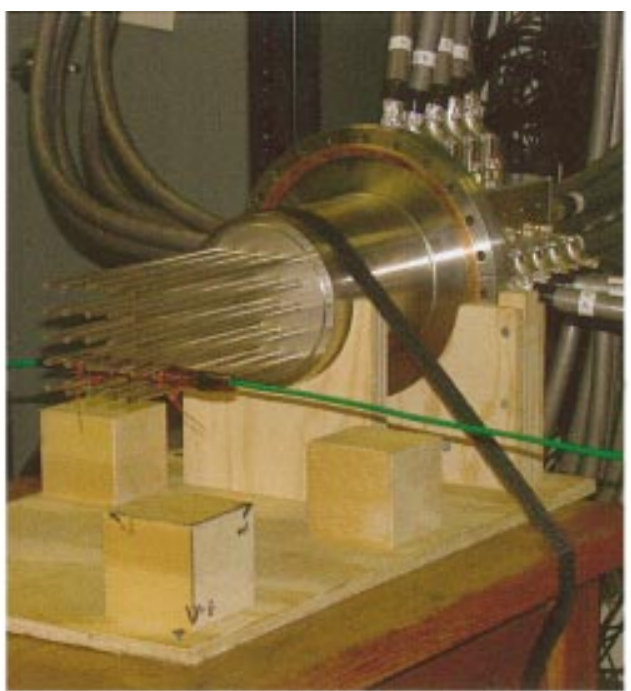

FIG. 8. (Color) A photograph (a) of the probe array during tests with a line current. (b) The measured magnetic field vectors (black) and field lines (blue and yellow), current density vectors (red), and magnetic energy density isosurfaces computed from them.

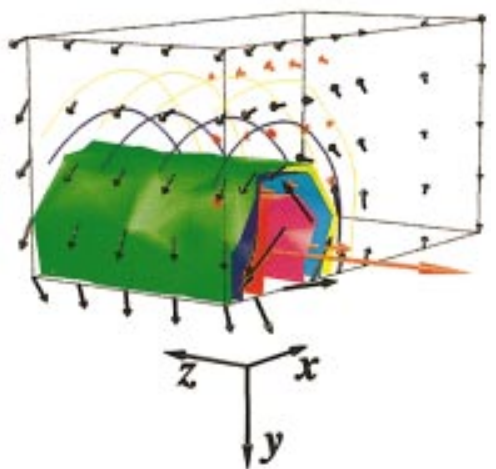

(b) surements are determined by the bit error in the digitization of the raw signals.

Finally, a test field from a line current was also used to evaluate the performance of the fully calibrated probe array. A more or less straight $2 \mathrm{~m}$ section of a large $10 \mathrm{~m}$ loop of wire was run through the probe array, as shown in Fig. 8(a), and driven with the pulsed-power bank used for the Helmholtz pair. Locally, therefore, the probe array measured approximately the field of an infinite line current with modest $(<10 \%)$ corrections from the remainder of the loop. Visually, Fig. 8(b) shows that the measured vector field matches the expected field of a line current. Field lines, current density, and magnetic energy density isosurfaces are computed from this data using a package of software tools developed in IDL.

Analytically, the measured data can be fit to the field of an infinite line current $\left(\mu_{0} I / 2 \pi r\right)$ with the magnitude, location, and direction of the current allowed to be free parameters. At several times during one of these line current shots, Fig. 9(a) compares the values for the magnitude of the current returned from the fitting procedure with the Rogowski coil measurement of the current in the wire. The agreement is within the anticipated errors for all shots. The positions and directions returned from these fits also correspond to the placement of the wire in the probe array, as expected.

In addition to fitting the measured magnetic fields, the current density computed on the lattice from $\mathbf{J}=\boldsymbol{\nabla} \times \mathbf{B} / \mu_{0}$ can be integrated over planes intersected by the wire (for example, $x y$ planes in Fig. 8) and compared to the total measured current in the wire. This is not as detailed of a check as the fitting procedure since Stokes' theorem reduces the calculation to a line integral of the fields on perimeter of each plane. Figure 9(b) shows this comparison at several times during a line current shot. Again, the results are within the anticipated errors.

\section{DISCUSSION}

General purpose massive data acquisition can be performed inexpensively and accurately using custom built mul- tiplexing electronics. This article describes one such system and its application for a 3D magnetic probe array at SSX. A similar multiplexing data acquisition system has also been implemented for several other applications at RPPL. Careful


FIG. 9. Comparison of the current measured with a Rogowski coil for a line current shot with the value obtained (a) by fitting the measured vector field to the field of an infinite line current and (b) by integrating the computed current density. 
control of the timing across the whole system is found to be the most stringent requirement for successful operation. The fully calibrated 3D probe array and data acquisition system can perform 600 measurements on up to $\pm 3 \mathrm{kG}$ fields with an accuracy of about $20 \mathrm{G}$ every $800 \mathrm{~ns}$. These results are robust under error analysis and cross checks with test fields. Experimental studies are now underway at SSX to measure the 3D structure of magnetic reconnection in merging spheromaks. The 3D probe array measurements allow detailed visualization of magnetic fields and their evolving structure at MHD time scales without averaging.

\section{ACKNOWLEDGMENTS}

Contributions to this project from Tom Kornack and Steve Palmer (Swarthmore College) are gratefully acknowledged.
${ }^{1}$ D. McLoskey, D. J. S. Birch, A. Sanderson, and K. Suhling, Rev. Sci. Instrum. 67, 2228 (1996).

${ }^{2}$ J. E. Bailey, R. Adams, A. L. Carlson, C. H. Ching, and A. B. Filuk, Rev. Sci. Instrum. 68, 1009 (1997).

${ }^{3}$ M. R. Brown, Phys. Plasmas 6, 1717 (1999).

${ }^{4}$ E. R. Priest and T. G. Forbes, Magnetic Reconnection (Cambridge University Press, Cambridge, 2000).

${ }^{5}$ E. N. Parker, Cosmical Magnetic Fields: Their Origin and Activity (Oxford University Press, Oxford, U.K., 1979).

${ }^{6}$ R. L. Stenzel and W. Gekelman, Phys. Rev. Lett. 42, 1055 (1979).

${ }^{7}$ M. Yamada et al., Phys. Rev. Lett. 78, 3117 (1997).

${ }^{8}$ R. L. Stenzel and W. Gekelman, J. Geophys. Res. 86, 649 (1981).

${ }^{9}$ M. Yamada et al., Phys. Rev. Lett. 65, 721 (1990).

${ }^{10}$ Y. Ono et al., Phys. Rev. Lett. 76, 3328 (1996).

${ }^{11}$ M. A. Shay et al., J. Geophys. Res. 103, 9165 (1998).

${ }^{12}$ D. Biskamp, E. Schwarz, and J. F. Drake, Phys. Rev. Lett. 75, 3850 (1995).

${ }^{13}$ W. Gekelman and H. Pfister, Phys. Fluids 31, 2017 (1988).

${ }^{14}$ Prototron Circuits, Redmond, WA.

${ }^{15}$ Prism Electronics, Redmond, WA. 